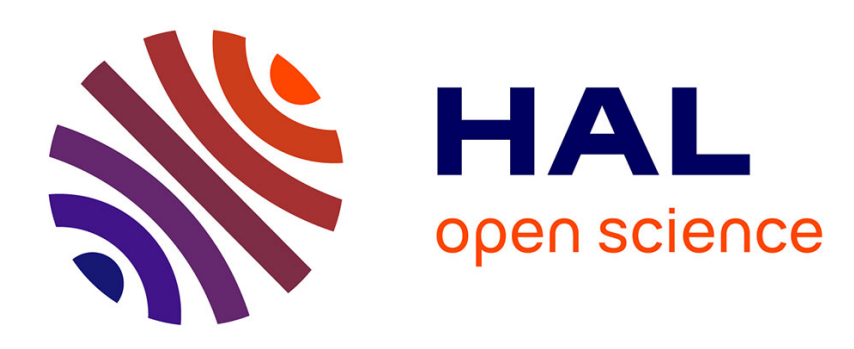

\title{
3He Experiments: Insights into Cosmology and Atomic Physics
}

Yuriy M. Bunkov

\section{To cite this version:}

Yuriy M. Bunkov. 3He Experiments: Insights into Cosmology and Atomic Physics. Journal of Low Temperature Physics, 2010, 158, pp.118-128. 10.1007/S10909-009-9997-5 . hal-00995199

\section{HAL Id: hal-00995199 \\ https://hal.science/hal-00995199}

Submitted on 21 May 2021

HAL is a multi-disciplinary open access archive for the deposit and dissemination of scientific research documents, whether they are published or not. The documents may come from teaching and research institutions in France or abroad, or from public or private research centers.
L'archive ouverte pluridisciplinaire HAL, est destinée au dépôt et à la diffusion de documents scientifiques de niveau recherche, publiés ou non, émanant des établissements d'enseignement et de recherche français ou étrangers, des laboratoires publics ou privés. 


\title{
${ }^{3}$ He Experiments: Insights into Cosmology and Atomic Physics
}

\author{
Y.M. Bunkov
}

Received: 21 July 2009 / Accepted: 29 September 2009 / Published online: 10 October 2009

(C) Springer Science+Business Media, LLC 2009

\begin{abstract}
Superfluid ${ }^{3} \mathrm{He}$, since its discovery by Osheroff, Richardson and Lee in 1972, has become one of the main systems for experimental studies of quantum field theories. This is due to the very rich order parameter of the quantum state for the triplet Cooper pairing of superfluid ${ }^{3} \mathrm{He}$, which exhibits, in addition to superfluid properties, properties of a magnetically-ordered quantum liquid crystal! The superfluid state, particularly at very low temperatures, is a quantum vacuum with a complicated order parameter, which carries various types of quasiparticles and topological defects. It can be considered a test system for the experimental investigations of many general physical problems in cosmology, atomic and nuclear physics, which are otherwise difficult or even impossible to investigate experimentally.
\end{abstract}

Keywords General physics · Cosmology · Bose-Einstein condensation · Superfluid ${ }^{3} \mathrm{He}$

PACS $67.57 . F g \cdot 05.45 . \mathrm{Yv} \cdot 11.27 .+\mathrm{d}$

\section{Introduction}

In this article I have space only to describe two types of experiment; the analogy between superfluid ${ }^{3} \mathrm{He}$ and the Universe during fast symmetry-breaking transitions, and the Bose-Einstein condensation of magnons.

Y.M. Bunkov (凶)

MCBT, Institute Neel, CNRS/UJF, Grenoble, 38042, France

e-mail: yuriy.bunkov@grenoble.cnrs.fr

Y.M. Bunkov

Kazan State University, 420008 Kazan, Russia 
The superfluid transitions in ${ }^{3} \mathrm{He}$ are characterized by broken gauge, spin and orbital rotation symmetries, and show many similarities to the unification and electroweak transitions in the Universe. One of the standing problems of cosmology is the dynamics of fast transitions after the Big Bang. In particular, one of the problems is the formation of cosmic strings. According to the theory of Kibble [1], rapid secondorder phase transitions in the Universe should create cosmic strings (vortices). The modification of this theory by Zurek [2], can be applied to transitions in other systems, particularly in superfluid liquids. We are able to test this theory in ${ }^{3} \mathrm{He}$. We can locally heat the superfluid ${ }^{3} \mathrm{He}$ by the nuclear reaction of neutron capture by the ${ }^{3} \mathrm{He}$ nucleus, which deposits $764 \mathrm{keV}$ of energy into the liquid. This energy heats a micron-sized region of the superfluid ${ }^{3} \mathrm{He}$ into the normal state. The region expands and then cools very rapidly through the transition in analogy with the behavior of the early universe after the Big Bang. It has been found experimentally in Grenoble [3] that a tangle of vortices forms after the fast phase transition in quantitative agreement with the Kibble-Zurek theory of cosmic-string formation. The analogy between superfluid ${ }^{3} \mathrm{He}$ and the Universe can be very deep. There are several superfluid phases of ${ }^{3} \mathrm{He}-\mathrm{A}, \mathrm{B}$ and $\mathrm{A} 1$. According to Linde [4], various states of the vacuum of the Universe may also exist. Studies of the transition between the various phases of superfluid ${ }^{3} \mathrm{He}$ can shed light on these profound properties of our Universe. In recent years we have studied the interaction of superfluid ${ }^{3} \mathrm{He}$ with muon, gamma and neutron radiation and confirmed the result of our pioneering experiments.

\section{Magnon BEC}

One of the problems I would like to highlight here is the Bose-Einstein condensation of magnons. The BEC state of magnons manifests itself in superfluid ${ }^{3} \mathrm{He}-\mathrm{B}$ by a long-lived induction signal (LLIDS) with particular non-linear properties [5, 6]. Previously, the (LLIDS) of small amplitude was observed in the case of the formation of stationary spin waves in a spin wave resonator [7, 8]. Non-linear behavior was not observed in this case. The BEC state has been observed in bulk superfluid ${ }^{3} \mathrm{He}$ in the presence of a large magnetic field gradient which plays the role of a magnetic trap for the BEC state. An NMR pulse NMR creates a large number of magnons, which precess at the local Larmor frequency. The induction signal decays very rapidly due to the inhomogeneity of the magnetic field. However, spin supercurrents, arising from the phase gradient generated by the precession, redistribute the non-equilibrium magnon gas. Finally the magnons become concentrated in the region of low magnetic field and form a domain with coherent precession, the Homogeneously Precessing Domain (HPD). The dipole-dipole frequency shift compensates the gradient of the field inside the domain. This effect is the magnetic analogy of the Meissner effect in superconductors. The HPD shows all the properties of a condensed matter superfluid state and can be considered as a "Spin Superfluid" state. Long-range spin supercurrent transport of magnetization has been observed in this state. It is important to note that the spin supercurrent is completely independent of any mass supercurrents. It is the property of the magnetic subsystem and can be found in the other magnetically 
Fig. 1 (Color online) The instantaneous spectrum of the HPD induction signal immediately after magnon excitation and after $0.03,0.3$ and $0.4 \mathrm{~s}$
Magnon Distribution

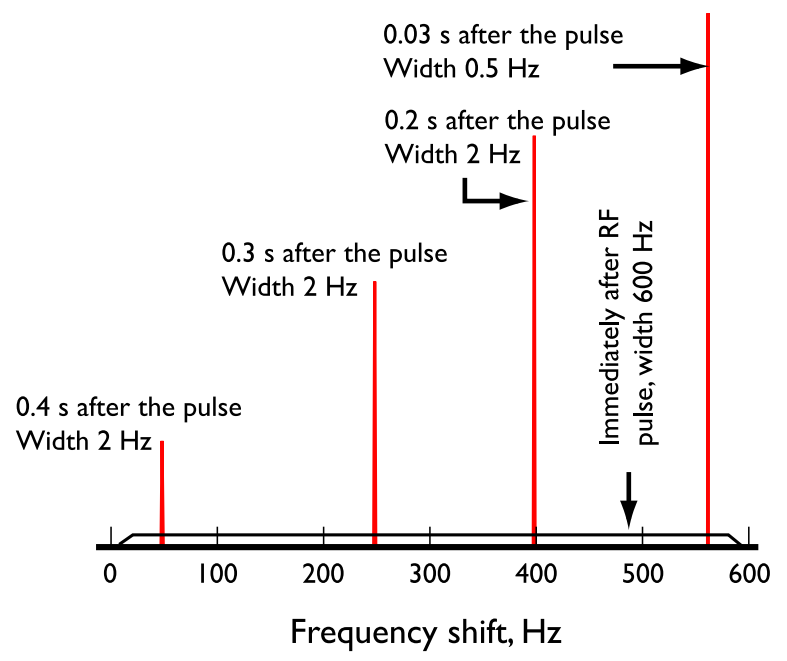

ordered materials with appropriate magnetic anisotropy. Anyway, the spin supercurrent in superfluid ${ }^{3} \mathrm{He}$ can also be regarded as a counterflow of two mass superfluid liquids with spin up and spin down.

Many spin superfluid phenomena have also been observed: spin-current Josephson effect; phase-slip processes at the critical current; and a spin current vortex-a topological defect which is the analog of a quantized vortex in superfluids and of an Abrikosov vortex in superconductors; Goldstone modes of HPD state etc. (see review [9], and references therein).

Finally, the question arises, is the magnon superfluidity related to Bose-Einstein condensation? The answer is, yes. It is possible to formulate the formation of the HPD in the language of Bose-Einstein Condensation [10]. Indeed, a droplet of magnon $\mathrm{BEC}$ can live in experiments up to $1 \mathrm{~s}$. This time is comparable with the lifetime of the droplet of atomic BEC! Furthermore, the fraction of BEC magnons can be almost $100 \%$. It is illustrated in Fig. 1. Under the conditions of the experiment, immediately after the NMR pulse the spectrum of the magnons is distributed over a frequency range of about $600 \mathrm{~Hz}$. Over a period of $30 \mathrm{~ms}$ the spectrum shrinks to only $0.5 \mathrm{~Hz}$ ! This is a factor of a 1000 condensation! It was shown [10] that the critical BEC temperature for the magnon gas density obtained in the experiments, is about $1 \mathrm{~K}$, while the experiments were performed at a temperature of about $1 \mathrm{mK}$. This temperatures ratio explains the near $100 \%$ condensation of the magnon gas. The number of non-equilibrium magnons remains practically the same during the time of formation of the BEC. Subsequently the density decreases due to relaxation. The frequency also slowly changes since the state slowly changes its position in the magnetic trap.

At very low temperatures when dissipation processes are virtually zero, a selfadjustment process of the spin-orbit interaction leads to the formation of a " $Q$ " ball which radiates a "Persistent" NMR signal $[11,12]$. This magnon-condensation mechanism is analogous to the BEC of dilute atomic gases in a trap, which is provided by 
the specific shape of the orbital field texture. A lifetime for this magnon BEC state of about one hour has been observed, much longer than any atomic BEC state! The "Q" ball provides a formal analogy with the mechanism of particle formation, suggested by Colleman [13].

For atomic gases we have only a one type of interaction. In the case of magnons in ${ }^{3} \mathrm{He}$ the interaction potential can be very different for different superfluid phases and order parameter orientations. The interaction can be attractive, as in bulk ${ }^{3} \mathrm{He}$ $\mathrm{A}$, or repulsive, as in ${ }^{3} \mathrm{He}-\mathrm{A}$ oriented by squeezed aerogel, or even very exotic, as in the case of ${ }^{3} \mathrm{He}-\mathrm{B}$. Finally, six different types of magnon BEC have been found in the superfluid phases of ${ }^{3} \mathrm{He}$ under different conditions. We can conclude that the phenomenon of the BEC of magnons exhibits very rich properties in comparison with an atomic BEC.

There have been many speculations on the BEC of magnons in magneticallyordered materials. In some magnetic systems, the symmetry-breaking phase transition starts when the system becomes softly unstable towards growth of one of the magnon modes. The condensation of this mode leads finally to the formation of the true equilibrium ordered state. In the same manner, the Bose condensation of phonon modes may serve as a soft mechanism for the formation of the equilibrium solid crystals. But this does not mean that the final crystal state is a Bose condensate of phonon. On the contrary, the BEC of quasiparticles is in principle a non-equilibrium phenomenon, since the quasiparticles (magnons) have a finite lifetime. In our case, the magnons live long enough to form a state very close to a thermodynamically equilibrium BEC, but still it is not an equilibrium. Ordered magnetic states are static equilibrium states with diagonal long-range order. The magnon BEC is a dynamic state characterized by off-diagonal long-range order, which is the principal signature of spin superfluidity [14].

\section{The Universe and ${ }^{3} \mathrm{He}$}

It is generally supposed that the universe was created by the "Big Bang", and that the early Universe cooled through a cascade of symmetry-breaking phase transitions accompanied by inflation. These transitions may have produced many types of topological defects: domain walls, cosmic strings (vortices) and monopoles $[1,15]$. The latest super-symmetry scenarios suggest that the initial symmetry of the Universe was $S O(10)$. After a cascade of transitions, we have now a lower symmetry state $S U(3) \times S U(2) \times U(1)$. A number of other low-symmetry states could have been produced during these transitions. It was shown that the ground state energy of these states lies between the energy of the $S U(3) \times S U(2) \times U(1)$ state and the $S U(4) \times U(1)$ state [4]. From this point of view the Universe is very similar to superfluid ${ }^{3} \mathrm{He}$, where different states (phases) can be found. The question of transitions between different states in ${ }^{3} \mathrm{He}$ is thus relevant for the Universe as will be discussed later.

Anyway, today we have our Universe, which is presented to us for investigation. There remain many paradoxes and questions to be answered. What we have now is a quantum vacuum populated with particles (bosons and fermions) combined to form 
atoms and molecules. Recently, humans succeeded in observing the long predicted Bose-Einstein condensation of atomic gases. Earlier, unpredicted coherent quantum states of matter had been discovered; superfluidity, and its electromagnetic analogue superconductivity and spin superfluidity. In the Universe, we also believe that we have non-barionic "Dark Matter" which does not interact with barionic matter by electromagnetic forces, although we can infer its existence through the gravitational interaction. There are currently many suggestions concerning the nature of "Dark Matter". The most popular candidates are a new class of supersymmetric particles known as WIMPs (Weak Interacting Massive Particles) and light particles known as AXIONS. The latter can exist in the form of a superfluid coherent quantum state. Finally, we have the further strange "Dark Energy" which accelerates the expansion of our Universe.

The Universe consists of a very complicated quantum vacuum with a large number of different types of excitations and, possibly, topological defects. The boundaries between different quantum vacua of the Universe (branes) are under discussion. Superfluid ${ }^{3} \mathrm{He}$ also consists of a very complicate quantum vacuum, with different types of excitations and topological defects. Even the boundaries between different vacua, such as between ${ }^{3} \mathrm{He}-\mathrm{A}$ and ${ }^{3} \mathrm{He}-\mathrm{B}$ or between two different orientations of order parameter ("cosmic defect") has been observed in superfluid ${ }^{3} \mathrm{He}$. Owing to the many similarities, superfluid ${ }^{3} \mathrm{He}$ can therefore play the role of a "test tube" for the Universe. Furthermore, for superfluid ${ }^{3} \mathrm{He}$ we already know the "theory of everything", which remains a dream for cosmology and High energy physicists!

In ${ }^{3} \mathrm{He}$ we can observe states with a) combined spin-orbit rotation symmetry $\left.\left({ }^{3} \mathrm{He}-\mathrm{B}\right), \mathrm{b}\right)$ two gauge symmetries $\left({ }^{3} \mathrm{He}-\mathrm{A}\right)$, and c) one gauge symmetry $\left({ }^{3} \mathrm{He}-\mathrm{A} 1\right)$. After the transition, superfluid ${ }^{3} \mathrm{He}$ can support many types of topological defects: monopoles, (known as boojums), various vortices (the analogs of cosmic strings) and two dimensional defects (analogs of branes). All these defects are shown schematically in Fig. 2. Various types of quasiparticle are also shown, the relics of normal ${ }^{3} \mathrm{He}$ (fermions); magnetic excitations from the ordered magnetic ground state, magnon (bosons) and various excitations of the orbital fields (analogous to the Higgs bosons), and the analogues of photons, and gravitons. I would like to emphasize here that the quasiparticles and magnons do not interact directly with each other but only through the orbital momentum! This is a very interesting new analogy. This behavior is like that between luminous matter and "Dark Matter", which interact only through gravity! Furthermore, magnons can form a Bose-Einstein condensed state. One of the hypothetical "Dark Matter" candidates, the Axion, can also possibly exist in a BEC state, which increases the difficultly of observing them directly in terrestrial experiments. The many examples of similarities make superfluid ${ }^{3} \mathrm{He}$ a very important material for 'experimental' studies of cosmology!

There is one additional very important similarity. A current hot topic in cosmology concerns the recently-discovered acceleration of the expansion of the Universe, with an all-pervading "Dark Energy" supposedly responsible. Is there a "Dark Energy" analogue in ${ }^{3} \mathrm{He}$ ? Yes! At relatively high temperatures and pressures the superfluid A phase is stable. However, the A phase can be cooled to lower temperatures where the $\mathrm{B}$ phase is stable and the A phase can only exist in a metastable state. When the transition is finally triggered at some point, the B phase expands into the region of 


\section{Droplet of Superfluid ${ }^{3} \mathrm{He}$.}

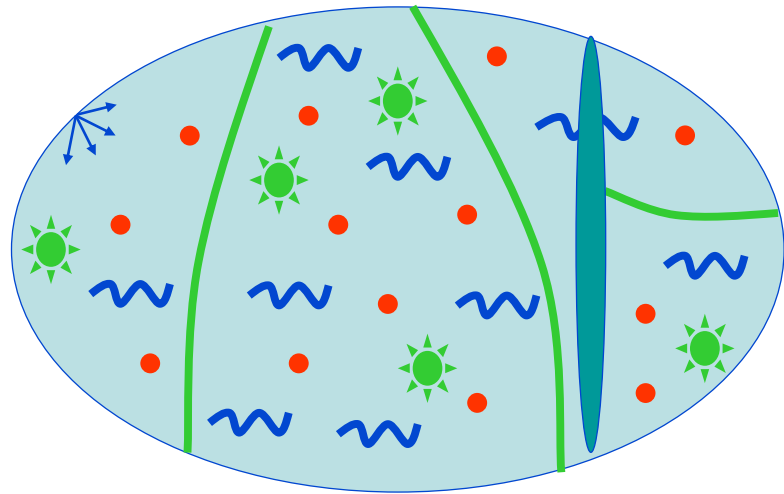

Particles:

Topological defects:
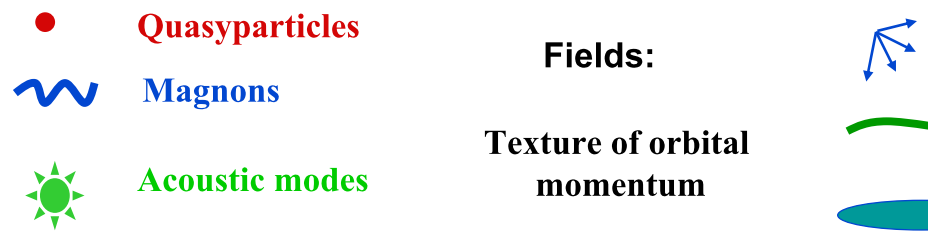

Boojum

Vortex

Brane

Fig. 2 (Color online) Particles, fields and topological defects in the superfluid ${ }^{3} \mathrm{He}$ world

the A phase. The difference between the chemical potentials of the A and B phases is the "Dark Energy" which drives the expansion of the B phase!

\section{Fast Transitions in ${ }^{3} \mathrm{He}$ and the Universe}

The conventional discussions of cosmological transitions in the Universe do not go beyond the simple model of a gauge transition with an energy profile in the shape of the "Mexican hat". In this case the order parameter is described by a single complex number or two Higgs fields with a single minima and a degenerate phase. In condensed matter this transition corresponds to ${ }^{4} \mathrm{He}$ superfluid transition or to the superconducting transition in conventional superconductors. The order parameter in ${ }^{3} \mathrm{He}$, however, is described by 9 complex numbers, giving a manifold of 18 dimensions. The hypothetical Universe $S U(5)$ symmetry can be represented by a 25 dimensional manifold. The multidimensional manifolds usually show an energy profile with a form much more complicated than the simple "Mexican hat" profile. There can be many local minima and saddle points; each corresponding to different states. In order to illustrate this, let us present graphically the potential as a deformed two dimensional "Mexican hat", as shown in Fig. 3.

At the transition the vacuum state moves downhill from the central maximum (false vacuum). The direction of this movement is determined by some small initial 
Fig. 3 (Color online)

A schematic 2D representation of the energy asymmetry of the 18D manifold of the superfluid ${ }^{3} \mathrm{He}$ order parameter. Vacuum B has a lower energy but a smaller probability of creation
Fig. 4 (Color online) The probability of nucleation of the A phase, and the energy difference between the $\mathrm{A}$ and $\mathrm{B}$ phases, as a function of pressure for temperatures near $T_{c}$

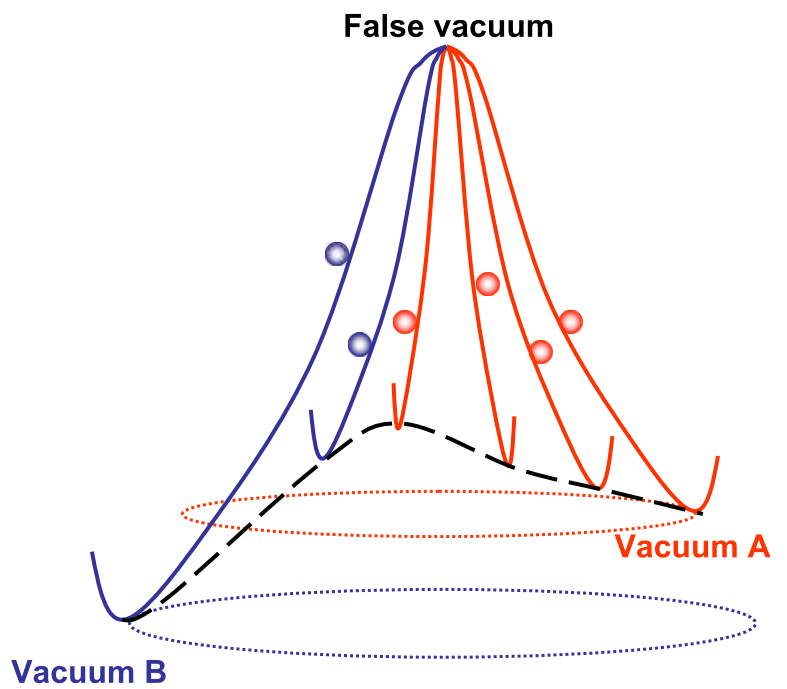

Vacuum B

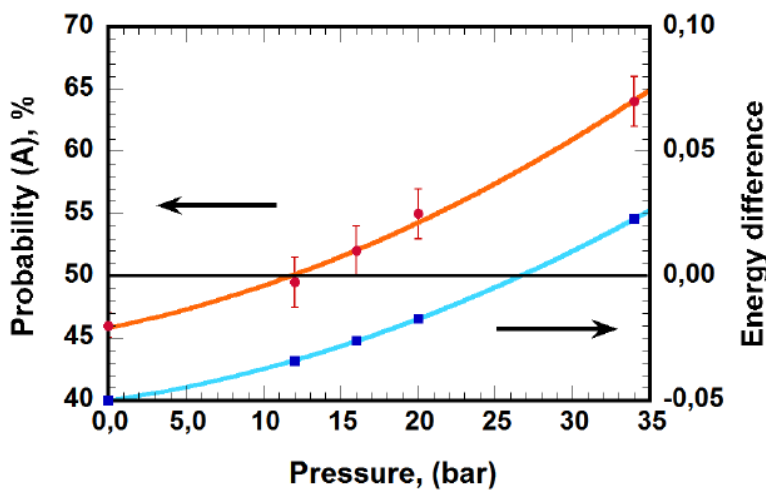

perturbation, which has an equal probability for any of the quantities in the orderparameter matrix. However, since the energy-potential profile is not symmetric, the probabilities of dropping into the A phase or into B phase are different and furthermore not determined by the ground-state energy but by the asymmetry of the profile near the high-symmetry state (false vacuum).

We know practically nothing about the energy profile for corresponding cosmological transitions. However, for the superfluid transition in ${ }^{3} \mathrm{He}$ we know the energy profile, at least in the Ginzburg-Landau approximation near $T_{c}$. On the basis of this energy profile, there has been a Monte-Carlo calculation of the nucleation probability for the different phases in ${ }^{3} \mathrm{He}$ near $T_{c}$ [16]. It was found that the probability strongly depends on the pressure, as shown in Fig. 4. In the same figure, the difference in energies between the A and B phases is also shown. The main result of this calculation is that the pressure at which the two energies are equivalent is significantly different from that for the same nucleation probability. This observation allows us to suggest a new scenario for the A-B phase transition relevant for the Universe. 
First of all let us discuss the application of the Kibble-Zurek scenario to the superfluid ${ }^{3} \mathrm{He}$ transition. During a fast transition, superfluid regions nucleate independently in causally-independent regions. During subsequent cooling these regions grow and touch each other. However, the phase of the wave function in these regions can match each other with a probability of only about $50 \%$. If there is no match, then a topological defect appears. Using an equation suggested by Zurek [2], one can calculate the density of the vortex tangle created for a given cooling rate. In Grenoble we have measured the balance of energy after a neutron capture reaction in ${ }^{3} \mathrm{He}$ and found that the missing deposited energy corresponds quantitatively to that retained in the vortex tangle. This experiment showed that the Kibble-Zurek theory works well, at least for a fast transition in superfluid ${ }^{3} \mathrm{He}$ at low pressure.

The Kibble-Zurek scenario was developed on the basis of the simple "Mexican hat" potential. What should be changed in the case of a manifold order parameter? Volovik [17] and Bunkov \& Timofeevskaya [16] suggested that the various causallyindependent regions can drop into different states! The consequences of these modifications for cosmology have not yet been discussed. For example, we do not know the probability of creating the vacuum of our Universe compared with that for creating possible alternative vacua. We are not even certain that our vacuum is not a metastable state! And if it is, how long other states might have survived after the transition. Can the expansion of the $S U(3) \times S U(2) \times U(1)$ vacuum into regions initially occupied by other vacua, be regarded as inflation? What about the "Dark Energy" which is observed in the Universe? Might it not just be the difference in energy between two low-lying states of the vacuum of the Universe, as it is in superfluid ${ }^{3} \mathrm{He}$ ? While we cannot provide answers, not knowing the energy profile for the multidimensional vacuum manifold of the Universe, we can apply these questions to superfluid ${ }^{3} \mathrm{He}$ itself.

\section{The A-B Phase Transition in ${ }^{3} \mathrm{He}$}

The nucleation of B phase from supercooled A phase remains an unsolved problem. Any obvious mechanism relying on homogeneous nucleation driven by thermal fluctuations gives nucleation rates which are literally hundreds of orders of magnitude lower than those observed experimentally. Leggett [18] suggested that the transition might be triggered by particle absorption which would overheat the ${ }^{3} \mathrm{He}$ locally. $\mathrm{He}$ also developed a theoretical scenario, the "Baked Alaska" model [19] in which he supposed that the B-phase seed expands to reach the critical radius $R_{c}$ while shielded from the outside A phase by a surrounding shell of normal ${ }^{3} \mathrm{He}$. From our point of view, this mechanism is very artificial.

Experimentally the A-B phase transition has been studied systematically in Helsinki [20]. Their results showed a "catastrophic line" at about $0.67 T_{c}$ : a narrow range of temperatures in which the B phase always nucleated. They interpreted their data as being in conflict with the "Backed Alaska" model. Systematic Stanford experiments [21] demonstrated the importance of radiation for nucleating the A-B transition. The experimental probability of nucleating the A-B transition as a function of neutron or gamma radiation and temperature has been fitted by the theoretical 
Fig. 5 A schematic representation of the $\mathrm{A}$ and $\mathrm{B}$ phase seeds which appear after diffusion cooling of the hot spot

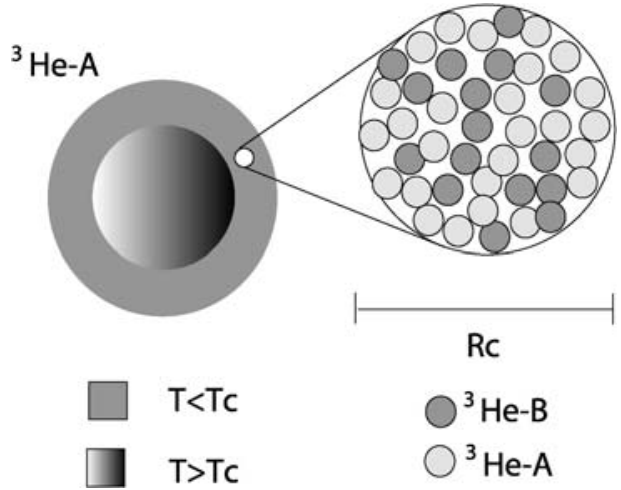

expression below, suggested for the "Baked Alaska" model:

$$
\tau=C \exp \left[a\left(R_{c} / R_{0}\right)^{n}\right],
$$

where $R_{0}$ is the value of $R_{c}$ at low temperature and magnetic field. The value for $n$ was estimated from the theory to lie between 3 and 5 . However, the fitting gives a value for $n$ equal to 1.5 ! This factor of two difference in the exponents was claimed in [21] to be experimental agreement with the theory! From our point of view, it shows only a lack of theory to explain the experiment. Furthermore, the "Baked Alaska" scenario has an internal problem. It does not take into account the balance of energies of the whole ensemble of excited quasiparticles inside the hot spot. There is NOT enough phase space to distribute all the quasiparticles within the region of a shielding shell [22].

Importantly, we do not need a normal shell to protect the interior of the hot bubble from the influence of the external A phase during the fast cooling. It has been shown in [23] that the diffusion temperature front moves so fast that the A-phase lags far behind and seeds of new states appear independently of the surrounding ${ }^{3} \mathrm{He}$ state, as shown in Fig. 5. After the neutron capture reaction, the size of the hot spot $\left(R_{h}\right)$ is considerably larger than the critical radius for $\mathrm{B}$ phase nucleation $\left(R_{c}\right)$. Thus there will be a huge number of seeds of both $\mathrm{A}$ and $\mathrm{B}$ phases nucleated inside the hot spot. On our estimations, at $29 \mathrm{bar}, R_{h}=30 \mu \mathrm{m}$ and $R_{0}=0.5 \mu \mathrm{m}$, while the radius of independently created seeds is about $R_{s}=0.07 \mu \mathrm{m}$.

We can suggest that the subsequent evolution of the very complicated mixture of A and B phases is determined, first, by the relative densities of the seeds of the two phases, secondly by the surface energy of the seeds' domain boundaries, and only thirdly by the energy balance between the phases. Computer calculations published in [16] play an important role in the understanding of the A-B transition. They show that the point where there is equal probability of nucleating the two phases does not correspond to the point at which the two phases have the same internal energies, but is significantly shifted to lower pressures, as shown in Fig. 4. This is the reason for the asymmetry in the A-B transition. When the B phase is energetically preferable, but the A phase has a higher probability of nucleation, then the A-phase seeds percolate inside the hot spot. Consequently B-phase seeds disappear owing to the A-B surface tension. In order to complete the transition, the B-phase seeds must percolate over 
a distance comparable to the critical cluster dimension. This is possible when the number of $\mathrm{B}$ seeds in a region of size $R_{c}$ is greater than the number of A seeds. There are two parameters that play an important role in these conditions: the probability of B-seed creation and the size of $R_{c}$.

In order to compare the predictions of the new scenario with experimental results, we need to know the probability of A- and B-phase creation at low temperature. Unfortunately, we only know the probability of the A and B seed formation near $T_{c}$. However, we can make an estimate for lower temperatures from the ${ }^{3} \mathrm{He}$ phase diagram. Were we to draw a line of equal probability parallel to the equilibrium line of the A-B transition in the phase diagram, we would delineate precisely the region where the A-B transition is triggered according to the measurements published in [20]. In order to get a quantitative agreement with the probability of the A-B transition measured experimentally at Stanford, we should suggest that the probability of the A-B transition after a neutron impact corresponds to the probability of the correlated formation of about 50 seeds of B phase. This means that the transition will trigger when about $50 \mathrm{~B}$-phase seeds are created within a short distance of each other. It is difficult to envisage the dynamics of the interaction between the various seeds after the transition. Nevertheless, the correlation of 50 seeds appears a reasonable result, since it makes up a large fraction of B seeds inside an $R_{c}$ volume, allowing the percolation of the B phase inside $R_{c}$ to trigger the transition.

Finally, this scenario (previously known as the "Aurora de Venice" but perhaps better renamed the "Cosmological" scenario) is a natural explanation of the A-B transition in superfluid ${ }^{3} \mathrm{He}$ on the basis of the well-established Kibble-Zurek scenario, without the need for any further artificial assumptions. Some criticisms of this theory, appear, particularly in [24] to be based mainly on a lack of understanding of our basic arguments.

\section{Conclusion}

In conclusion, we find that experimental studies of the superfluid states of ${ }^{3} \mathrm{He}$, particularly at the limit of the lowest possible temperatures, can reformulate and provide fruitful ideas for a solving similar problems in the physics of other complex quantum systems such as cosmology and atomic and high-energy physics.

\section{References}

1. T.W.B. Kibble, J. Phys. A 9, 1387 (1976)

2. W.H. Zurek, Nature 317, 505 (1985)

3. C. Bäuerle, Yu.M. Bunkov, S.N. Fisher et al., Nature 382, 332 (1996)

4. A. Linde, Particle Physics and Inflationary Cosmology (Harwood Academic, Reading, 1990)

5. A.S. Borovik-Romanov, Yu.M. Bunkov, V.V. Dmitriev, et al., JETP Lett. 40, 1033 (1984)

6. I.A. Fomin, JETP Lett. 40, 1036 (1984)

7. L.R. Corruccini, D.D. Osheroff, Phys. Rev. B 17, 126 (1978)

8. R.W. Giannetta, E.N. Smith, D.M. Lee, J. Low Temp. Phys. 45, 295 (1981)

9. Yu.M. Bunkov, Spin supercurrent and novel properties of NMR in ${ }^{3} \mathrm{He}$, in Progress in Low Temp. Physics, ed. by W. Halperin, vol. 14 (Elsevier, Amsterdam, 1995), p. 69

10. Yu.M. Bunkov, G.E. Volovik, J. Low Temp. Phys. 150, 135 (2008) 
11. Yu.M. Bunkov et al., Phys. Rev. Lett. 69, 3092 (1992)

12. D.I. Bradley et al., J. Low Temp. Phys. 134, 351 (2004)

13. Yu.M. Bunkov, G.E. Volovik, Phys. Rev. Lett. 98, 265302 (2007)

14. Yu.M. Bunkov, G.E. Volovik, arXiv:0904.3889 (2009)

15. Ya.B. Zel'dovich, I.Ya. Kobzarev, L.B. Okun', Sov. Phys. JETP 40, 1 (1975)

16. Yu.M. Bunkov, O.D. Timofeevskaya, Phys. Rev. Lett. 80, 4927 (1998)

17. G.E. Volovik, private communications (1997)

18. A.J. Leggett, Phys. Rev. Lett. 53, 1096 (1984)

19. A.J. Leggett, S.K. Yip, in Helium Three, ed. by V.M. Agranovich, A.A. Maradudin (North-Holland, Amsterdam, 1990), pp. 523-608

20. P.J. Hakonen et al., Phys. Rev. Lett. 54, 245 (1985)

21. P. Schiffer, D.D. Osheroff, A.J. Leggett, in Progress in Low Temp. Physics, ed. by W. Halperin, vol. 14 (Elsevier, Amsterdam, 1995), pp. 159-211

22. Yu.M. Bunkov, O.D. Timofeevskaya, Phys. Rev. Lett. 82, 3926 (1999)

23. T.W.B. Kibble, G.E. Volovik, JETP Lett. 65, 102 (1997)

24. A.J. Leggett, J. Low Temp. Phys. 126, 775 (2002) 